\title{
A change in scope: redefining minimally invasive
}

\author{
Tobias Stornebrink $^{1,2,3} \cdot$ Kaj S. Emanuel ${ }^{1,2,3,4} \cdot$ Yoshiharu Shimozono $^{5}$. Jón Karlsson ${ }^{6} \cdot$ John G. Kennedy ${ }^{5}$. \\ Gino M. M. J. Kerkhoffs ${ }^{1,2,3}$
}

Received: 15 December 2019 / Accepted: 3 February 2020 / Published online: 11 February 2020

(c) European Society of Sports Traumatology, Knee Surgery, Arthroscopy (ESSKA) 2020

The editors of KSSTA recently highlighted the increase in demand for ankle arthroscopy that is expected for the near future [5]. Arthroscopic techniques are increasingly sophisticated and the range of indications for arthroscopy is broadening accordingly. In contrast with these developments, arthroscopic cameras have hardly changed since their first introduction. We still use rod-lens scopes of substantial diameters, making arthroscopy as invasive as it was decades ago: posing risk to neurovascular tissue [3] and cartilage [4] and causing unnecessarily long recovery times. Current innovation in the field of needle arthroscopy might help to better facilitate the pending increase in ankle arthroscopic procedures.

In several lab and patient settings, our international collaborative group has been working with a novel needle arthroscopic system (NanoScope ${ }^{\mathrm{TM}}$, Arthrex, Naples, FL). With an optic chip at the camera tip and no inner rod-lenses, this semi-rigid scope remains durable at a total diameter of just $1.9-\mathrm{mm}$, and $2.26 \mathrm{~mm}$ including the cannula. Image quality is higher than that of traditional needle arthroscopes, offering a $400 \times 400$ pixel number with a $120^{\circ}$ field of view.

Gino M. M. J. Kerkhoffs

g.m.kerkhoffs@amsterdamumc.nl

1 Department of Orthopedic Surgery, Amsterdam Movement Sciences, Amsterdam UMC, University of Amsterdam, Meibergdreef 9, 1105 AZ Amsterdam, The Netherlands

2 Academic Center for Evidence Based Sports Medicine (ACES), Amsterdam, The Netherlands

3 Amsterdam Collaboration for Health and Safety in Sports (ACHSS), International Olympic Committee (IOC) Research Center Amsterdam UMC, Amsterdam, The Netherlands

4 Department of Orthopedic Surgery, Maastricht University Medical Center, Maastricht, The Netherlands

5 Department of Orthopedic Surgery, NYU Langone Health, New York, NY, USA

6 Department of Orthopaedics, Sahlgrenska University Hospital, Sahlgrenska Academy, Gothenburg University, Gothenburg, Sweden
In contrast with previous needle arthroscopes, this new system comes with tailored surgical instruments, which introduces the possibility of operative needle arthroscopy. The safety and efficacy of this technology was systematically tested in a cadaveric setting, and this fundamental research was translated into a broad clinical portfolio. The results of first studies are promising [1,2]. Cadaveric experience in the ankle is presented in this issue of KSSTA [1].

In general, operative needle arthroscopy offers a minimally invasive technique, with a less than 3-mm skin incision that requires only paper suture closure. This minimal portal footprint reduces local soft-tissue trauma and often obviates the need for any more than a local anaesthetic, both factors expediting patient recovery. Under these circumstances, smaller arthroscopic interventions can become bedside procedures performed in an office setting. Anterior and posterior ankle arthroscopy with debridement of osteophytes and cicatrized tissue is a reproducible technique that lends itself particularly well for operative needle arthroscopy. First metatarsal phalangeal joint limited cheilectomy and evaluation of chondral wear is another facile use. Tendoscopy of the Achilles, peroneal and tibialis posterior tendons is an attractive and simple procedure that can be both diagnostic and therapeutic in nature, allowing easy debridement using 2 and 3-mm shavers and augmenting repairs with biologic delivery systems. Similar ease of access can also be achieved in the knee, shoulder and other joints, where bony and soft-tissue impingement can be identified with clarity and addressed appropriately. Furthermore, clinical experience has confirmed the versatility of this technique as both a bona fide method of performing many therapeutic procedures as well as an augment to diagnostics.

The use of operative needle arthroscopy should not only be considered as an alternative to traditional arthroscopy alone, as it can often take a mere supportive role as well. Post-reduction visualization of intra-articular fractures is an obvious application in a trauma setting. Whilst obviating the need for high pressure fluid inflow systems it provides excellent visualization of intra-articular pathology, offering 
both a prognostic indicator as well as a means of delivering chondro-protective adjuncts if required. In addition, operative needle arthroscopy may fulfil an invaluable role in cartilage evaluation, both at the time of injury and during second look procedures after repair. It may assess the extent of damage and the quality as well as quantity of cartilage repair, a metric that MRI has some difficulty establishing [6].

In short, operative needle arthroscopy may fuel innovation in both diagnosis and treatment of intra-articular, tendon, and soft-tissue pathology. At this time, costs of the single use disposable system seem fair. Yet, formal evaluations of cost-effectiveness compared to traditional arthroscopy are being conducted. As long as the quality of imaging remains high and positive outcomes persist, operative needle arthroscopy may provide a new definition of minimally invasive and help us to better respond to the increased demand for arthroscopic care.

\section{References}

1. Stornebrink T, Altink JN, Appelt D, Wijdicks CA, Stufkens SA, Kerkhoffs GM (2020) Two-millimetre diameter operative arthroscopy of the ankle is safe and effective. Knee Surg Sports Traumatol Arthrosc. https://doi.org/10.1007/s00167-020-05889-7

2. Stornebrink T, Stufkens SA, Appelt D, Wijdicks C, Kerkhoffs GM (2019) 2-mm diameter operative tendoscopy of the tibialis posterior, peroneal and Achilles tendons is safe and effective: a cadaveric study of a minimally invasive approach to tendoscopy. Foot Ankle Int. https://doi.org/10.1177/1071100719895504

3. Vega J, Dalmau-Pastor M, Malagelada F, Fargues-Polo B, Pena F (2017) Ankle arthroscopy: an update. J Bone Jt Surg Am 99:1395-1407

4. Vega J, Golano P, Pena F (2016) Iatrogenic articular cartilage injuries during ankle arthroscopy. Knee Surg Sports Traumatol Arthrosc 24:1304-1310

5. Vega J, Karlsson J, Kerkhoffs GMMJ, Dalmau-Pastor M (2019) Ankle arthroscopy: the wave that's coming. Knee Surg Sports Traumatol Arthrosc. https://doi.org/10.1007/s00167-019-05813-8

6. Yasui Y, Hannon CP, Fraser EJ, Ackermann J, Boakye L, Ross KA et al (2019) Lesion size measured on MRI does not accurately reflect arthroscopic measurement in talar osteochondral lesions. Orthop J Sports Med. https://doi.org/10.1177/23259 67118825261

Publisher's Note Springer Nature remains neutral with regard to jurisdictional claims in published maps and institutional affiliations. 